\title{
Disappearance of Self-Focusing for Few-Cycle THz Pulses
}

\author{
$\underline{\text { S. A. Kozlov }}{ }^{1}$, A. A. Drozdov ${ }^{1}$, M. A. Kniazev ${ }^{1}$, D. A. Kislin ${ }^{1}$, S. Choudhary ${ }^{2,3}$, R. W. Boyd ${ }^{2,3}$ \\ ${ }^{1}$ ITMO University, Saint-Petersburg, Russia, kozlov@mail.ifmo.ru \\ ${ }^{2}$ University of Ottawa, Ottawa, Canada \\ ${ }^{3}$ The Institute of Optics, University of Rochester, Rochester, NY 14627-0186, USA
}

In the present work, we demonstrate that, for fewcycle wave packets with longitudinal dimension less the transverse size, the concept of critical power of self-focusing can lose its physical meaning because of the dominance of dispersion over diffraction. We present simple formulas for the estimation of the parameters of the field and medium under which selffocusing disappears and illustrate with numerical calculations the changes in the self-action phenomenon of light in this case.

\section{Theoretical estimations}

We have shown that when the nonlinearity of the optical medium competes primarily not with diffraction but with dispersion, the concept of critical power for self-focusing of radiation $P_{\mathrm{cr}}$ will begin to lose its original meaning. Such condition can be expressed in terms of laboratory parameters as:

$$
\frac{l_{0}}{D_{0}}<\sqrt{c \omega_{0} n\left(\omega_{0}\right) \beta_{2}},
$$

where $l_{0}=2 c \tau_{0}$ is longitudinal size of the wave packet and $D_{0}=2 r_{0}$ is its transverse size, $c$ is the speed of light in vacuum, $\tau_{0}$ and $r_{0}$ are the duration and radius of the beam at the entrance of nonlinear medium, $\omega_{0}$ is the central frequency of radiation, $n\left(\omega_{0}\right)$ is the linear refractive index of the medium at central frequency, and $\beta_{2}=\left(\partial^{2} k / \partial \omega^{2}\right)_{\omega_{0}}$, where $k$ is the wave number.

For an extremely simple dispersion formula of $\mathrm{n}(\omega)=N_{0}+a c \omega_{0}^{2}$, where $N_{0}$ and $a$ characterize the medium dispersion, the inequality (1) can be reduced to

$$
\frac{l_{0}}{D_{0}}<\sqrt{6 N_{0} \Delta n_{\text {disp }}},
$$

where $\Delta n_{\text {disp }}=a c \omega_{0}^{2}$ is the change of the refractive index at the central wavelength due to dispersion.

\section{Numerical illustrations}

We consider boundary conditions (the electric field of radiation at the entrance to nonlinear medium) in the form of a Gaussian axisymmetric paraxial beam with a small number of oscillations $\left(N=\tau_{0} / T_{0}\right)$

$$
E(0, r, t)=E_{0} \exp \left(-\frac{r^{2}}{r_{0}^{2}}\right) \exp \left(-\frac{t^{2}}{\tau_{0}^{2}}\right) \sin \left(\omega_{0} t\right),
$$

where $r=\sqrt{x^{2}+y^{2}}$, and $T_{0}=2 \pi / \omega_{0}$ is central period of electric field oscillations.

The pulse propagation was modeled in terms of the evolution of the instantaneous electric field $[1,2]$ and not its envelope. The use of the field equation allows on the correctly describe the generation of radiation at tripled and higher frequencies [1]. We show two-dimensional plots of the electric field distribution in Figures 1-3. The light-grey and dark-grey areas correspond to maximum positive and negative values of the electric field, while white lines correspond to zero field values, respectively.
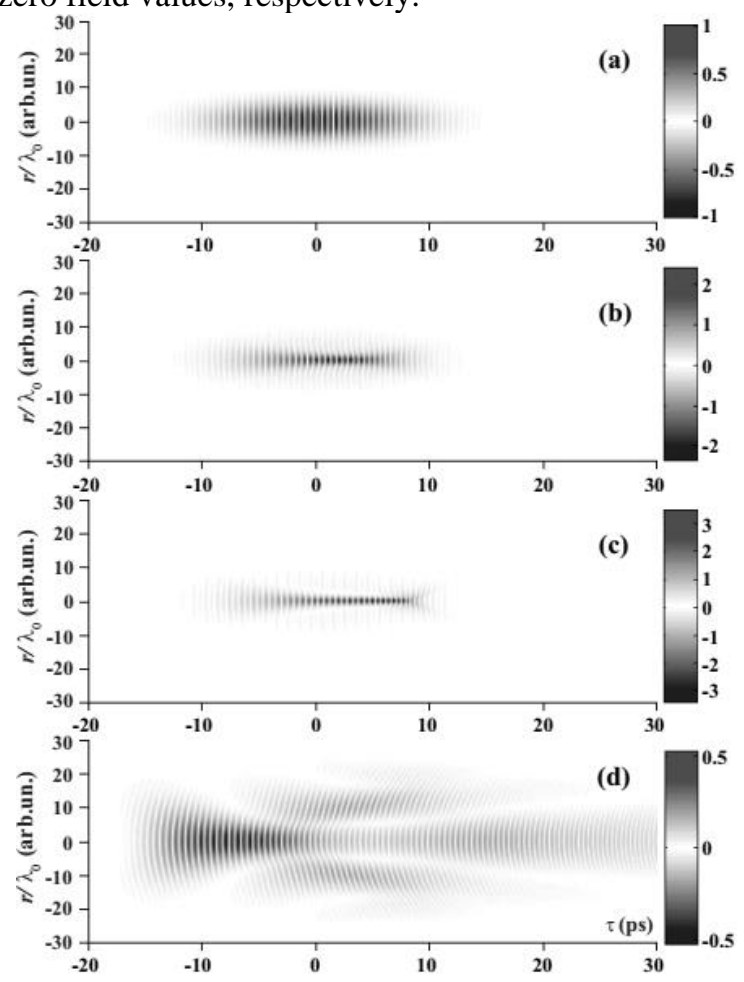

Fig. 1. The spatiotemporal dynamics of the electric $\mathrm{THz}$ field $E$ of wave packet (3) in a $\mathrm{MgO}: \mathrm{LiNbO}_{3}$ crystal at distances (a) $z=0 \mathrm{~cm}$, (b) $z=8 \mathrm{~cm}$, (c) $z=11 \mathrm{~cm}$, (d) $z=25$ $\mathrm{cm}$. Initial pulse parameters are $\lambda_{0}=300 \mu \mathrm{m}, r_{0}=5 \lambda_{0}, \tau_{0}=8$ ps, $I=3 \times 10^{8} \mathrm{~W} / \mathrm{cm}^{2}, P_{0}=4 P_{\text {cr. }}$ We see that the beam shows self-focusing behavior at 8 and $11 \mathrm{~cm}$ and the beginning of breakup into filaments at $25 \mathrm{~cm}$

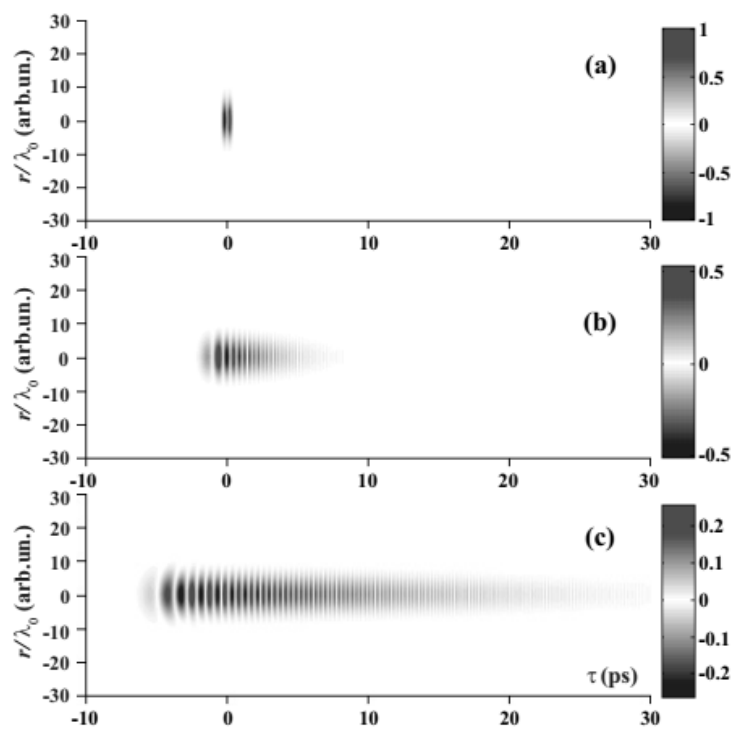


Fig. 2. The spatiotemporal dynamics of the electric field for a single-cycle $\mathrm{THz}$ wave packet (3) in a $\mathrm{MgO}: \mathrm{LiNbO}_{3}$ crystal at distances (a) $z=0 \mathrm{~cm}$, (b) $z=0.5 \mathrm{~cm}$, (c) $z=2 \mathrm{~cm}$. Initial pulse parameters are $\lambda_{0}=300 \mu \mathrm{m}, r_{0}=5 \lambda_{0}, \tau_{0}=0.3$ ps, $I=3 \times 10^{8} \mathrm{~W} / \mathrm{cm}^{2}, P_{0}=4 P_{\text {cr. }}$ In this case the beam does not display self-focusing

Figures 1 and 2 show typical results for the selffocusing dynamics for a many-cycle pulse (Fig 1) and a single-cycle pulse (Fig 2). The changes in selffocusing dynamics are illustrated for ultrashort pulses with a central wavelength of $\lambda_{0}=300 \mu \mathrm{m}$ for a number of field oscillations $N=16\left(2 \tau_{0}=16 \mathrm{ps}\right)$ and $N=$ $0.6\left(2 \tau_{0}=0.6 \mathrm{ps}\right)$. Stoichiometric $\mathrm{MgO}: \mathrm{LiNbO}_{3}$ crystal with nonlinear refractive index $n_{2}=5.4 \times 10^{-12}$ $\mathrm{cm}^{2} / \mathrm{W}$ [1] and dispersion parameters described in [1] was considered as the nonlinear dielectric medium. We also used the same ratio between initial power $P_{0}$ and critical power $P_{\text {cr }}$ for self-focusing, $P_{0}=4 P_{\text {cr. }}$ As can be seen from Fig. 1, at initially stage of $\mathrm{THz}$ wave propagation its transverse compression occurs (Fig. 1 b). Then, a shock wave is formed in the generated filament (Fig. $1 \mathrm{c}$ ). At the distance of $z=11 \mathrm{~cm}$ (Fig. $1 \mathrm{c})$ a self-focused filament is formed with a transverse size 6.5 times smaller than the input value. The intensity of the radiation increased by a factor of 12 within the filament and reaches a value of $3.6 \times 10^{9}$ $\mathrm{W} / \mathrm{cm}^{2}$. The on-axis pulse duration is seen to decrease by a factor of 1.4 at the $\mathrm{e}^{-1}$ level.

With a decreasing of the number of oscillations down to just one (Fig. 2), the dispersion becomes so strong that self-focusing is no longer observed. For example, the axial pulse duration increased in 13 times (at $\mathrm{e}^{-1}$ level) already at a distance of $z=5 \mathrm{~mm}$ (Fig. 2 b). The curvature of the wave front due to diffraction is noticeable only at the leading edge of the beam. As one can see from Figures 1-2, reduction of $N$ leads to decreasing of self-focusing efficiency because dispersion starts play a significant role in pulse evolution.

For a higher intensity of the input single-cycle $\mathrm{THz}$ beam (for example for $I=1.48 \times 10^{10} \mathrm{~W} / \mathrm{cm}^{2}$ such that $P_{0}=200 P_{\mathrm{cr}}$ ), we observe an effect that is similar to the phenomenon of self-focusing of radiation (Fig. 1). In contrast to the case considered in Fig. 1 , a significant increase of pulse duration is observed here at the initial stage of $\mathrm{THz}$ wave propagation because of the combined strong effect of dispersion and nonlinearity. Significant curvature of the wave front due to nonlinearity is also observed (Fig. 3 b,c). At the next step of $\mathrm{THz}$ wave propagation, transverse compression occurs due to the curvature. At the distance of $z=44 \mathrm{~mm}$ (Fig. $3 \mathrm{~d}$ ), a filament is formed with transverse dimension 6 times smaller than the input value. However, the intensity of the radiation is decreased down to $5.3 \times 10^{9} \mathrm{~W} / \mathrm{cm}^{2}$. In Fig. $3 \mathrm{~d}$ we show additionally the result of propagation of the initial beam (Fig. 3 c) in linear dielectric medium $20 \mathrm{~mm}$ long with the same dispersion. It can be clearly seen that the focusing occurs in a linear medium as well due to the large curvature of the phase surfaces. Then usual dispersive-diffraction wave packet spreading occurs (Fig. 3 e).

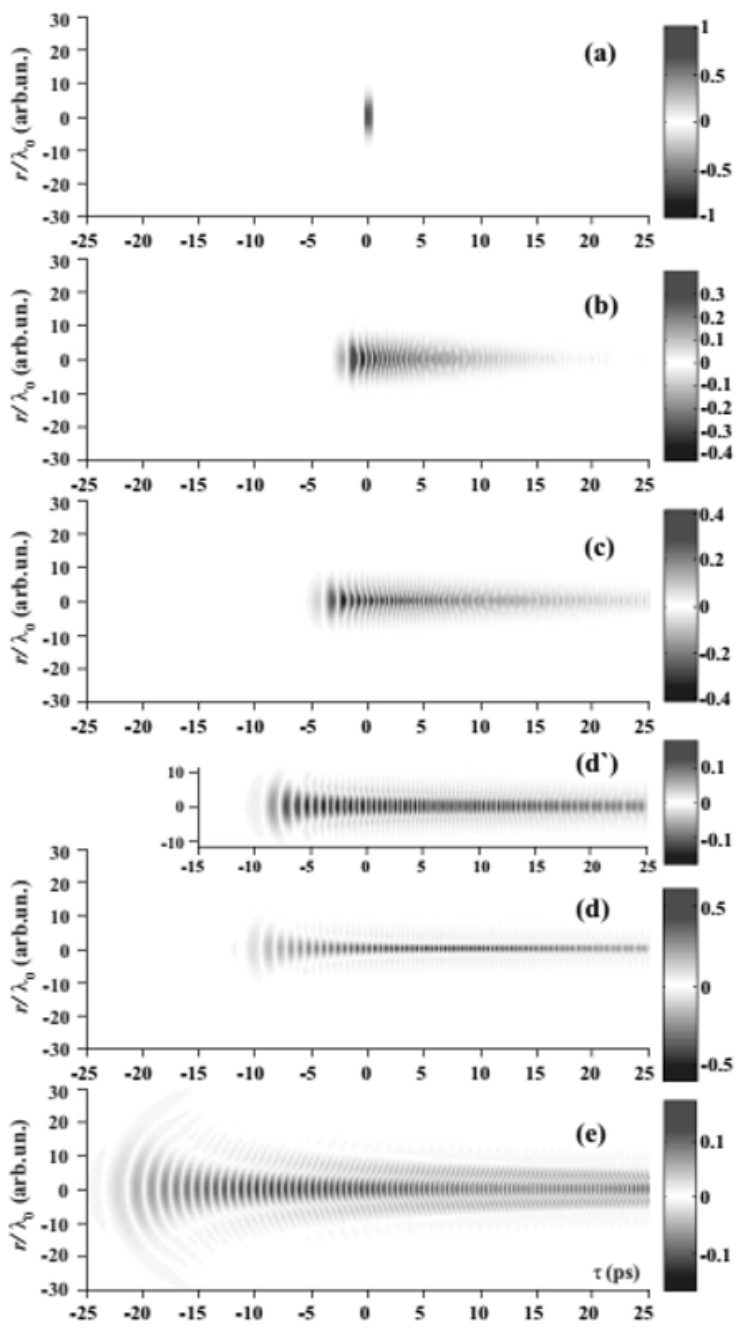

Fig. 3. The spatiotemporal dynamics of the $\mathrm{THz}$ electric field $E$ of a single-cycle wave packet (3) in a $\mathrm{MgO}: \mathrm{LiNbO}_{3}$ crystal at distances (a) $z=0$, (b) $z=9 \mathrm{~mm}$, (c) $z=17 \mathrm{~mm}$, (d) $z=44 \mathrm{~mm}$, (e) $z=90 \mathrm{~mm}$. Initial pulse parameters are $\lambda_{0}=300 \mu \mathrm{m}, r_{0}=5 \lambda_{0}, \tau_{0}=0.3 \mathrm{ps}, I=1.48 \times 10^{10} \mathrm{~W} / \mathrm{cm}^{2}$, $P_{0}=200 P_{\text {cr. }}$ Case $\left(\mathrm{d}^{\star}\right)$ corresponds to propagation of the initial beam though a $20-\mathrm{mm}$-long linear dielectric medium

\section{Conclusion}

A simple expression is derived and analyzed for the conditions under which the concept of a critical power for self-focusing loses its fundamental physical meaning due to the dominance of the process of dispersion over diffraction. It is shown that the power of single-cycle terahertz waves, when self-focusing occurs, can exceed the critical power by several hundred times. It is also shown that the nature of the compression of the single-cycle light beam is significantly different from the self-focusing of longer pulses.

\section{References}

1. Drozdov, A. A., Kozlov, S. A., Sukhorukov, A. A., Kivshar, $Y$. S. Self-phase modulation and frequency generation with few-cycle optical pulses in nonlinear dispersive media // Phys. Rev. A. 2012. V. 86, No. 5. P. 053822.

2. Kozlov, S. A., Samartsev, V. V. Fundamentals of femtosecond optics Woodhead. Cambridge. UK. 2013. P. 272. 